\title{
A Noble Gestures Control System Using Machine Learning
}

\author{
Jang Yeol Lee, Earl Kim, Jun Heon Kim, Choon Sung Nam, and Dong Ryeol Shin
}

\begin{abstract}
In this paper, we propose a method to improve the recognition rate of gestures motion and scalability problem which can occur in gestures when operate drone using machine learning. For these purposes, the gestures data transmitted from the drone controller are used for machine learning on real time, and a new learning model is periodically created using the gestures data stored in the HDFS(Hadoop Distributed File System). The goal of our proposed system is to increase the recognition rate of the gestures motion when new learning model is created. In addition, it is possible to expect enhanced scalability through recognition of gestures motion, and that drone is able to recognize a new gestures motion which is not defined in the server.
\end{abstract}

Keywords—Big Data, Apache Flume, HDFS, Apache Spark

\section{Introduction}

Worldwide, interest in drone continues to grow. The use of drone, usually studied in military use in past days, has been rapidly increased for commercial purpose and has been applied to various fields such as logistics delivery and information communication. As the drone industry develops grows and matures, Gesture drones are appearing decors such as smartphones and tablets. Gestures drone' gestures motion recognition rate depends on the controller. Although, on-the-shelf gesture controller has high recognition rate, it is difficult to provide $100 \%$ recognition rate [1].

gestures can only be used in a limited manner depending on the type of gestures pre-populated in the controller. This means that there is no way to add a new gestures action other than the gestures action set in the controller when the product is released. Therefore, the scalability of the gestures is difficult[2].

Jang Yeol Lee

Sungkyunkwan University

South Korea

Earl Kim

Sungkyunkwan University

South Korea

Jun Heon Kim

Sungkyunkwan University

South Korea

Choon Sung Nam

Sungkyunkwan University

South Korea

Dong Ryeol Shin

Sungkyunkwan University

South Korea
In this paper, we propose a drone gestures control system using machine learning. Gestures data is collected through MYO device using gyro-sensor and EMG(electromyogram) as a tool to measure gestures. The gesture controller sends commands to the drone using measured data. Moreover, we propose a method to improve gestures recognition rate by transmitting gestures data to machine learning server. When the machine learning server receives the gestures motion data from the controller, trains the existing model and then sends the newly trained model back to the controller the newly created model back to the controller. Therefore, the controller, reflects the newly created model, can have a better gestures recognition rate than before. We can also use the gestures controller to add new gestures behavior that was not previously available, also can configure the UI(User Interface) on the controller to add new actions, and use the newly added gestures to control the drone. The machine learning server executes the algorithm to reflect the new operation, learns the new gestures operation to the existing model, and sends it back to the controller. In this way, it is possible to increase the recognition rate of the gestures motion of the gestures drone and the extensibility of the gestures operation by adding a new gesture motion as well.

The composition of this paper is as follows. In Section 2 , we discuss related work on the machine learning server that constitutes the gestures drone system. In Chapter 3, we introduce and give specific description of the "gestures drone" machine learning system. In Chapter 4, we conclude with a review of future work.

\section{Related Work}

\section{A. Apache Flume}

Apache flume is a framework for data collection, which can collect various log data and transmit data in real time[3]. Apache flume is designed with the core of reliability, scalability and manageability of the system.

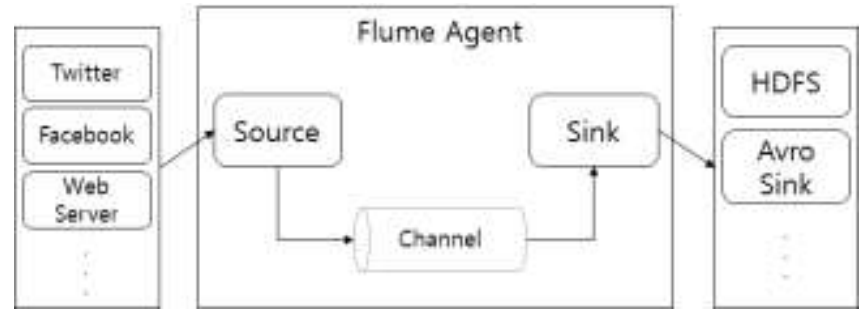

Figure 1. Apache flume dafa flow model

The Apache flume consists of source, channel, and sink as shown in Figure 1. The source collects logs from the server, and the channel serves as a temporary repository for delivery to the sink. The sink is responsible for storing the channel's data locally or in repositories such as HDFS (Hadoop Distributed File System) and Apache HBase. There 
are Avro type for connecting source to agent, NetCat for TCP communication, and syslog and Exec type. Channels store data received in one of three forms: Memory, JDBC, or File. Sink can transfer received data to HDFS, Apache HBase, File_Roll, or another agent and store it in the desired repository.

\section{B. Apache Spark}

Apache Spark is a distributed data analysis system that runs on memory[4]. Apache Spark supports a variety of languages including Java, Scala, Python, and R. Spark's strength is that Spark's MapReduce speed is superior when compared to Hadoop's MapReduce speed. Because Spark stores input, output, and intermediate data in RDD(Resilient Distributed Dataset) in memory, so it can quickly process interactive workloads and perform better without additional $\mathrm{I} / \mathrm{O}$ costs

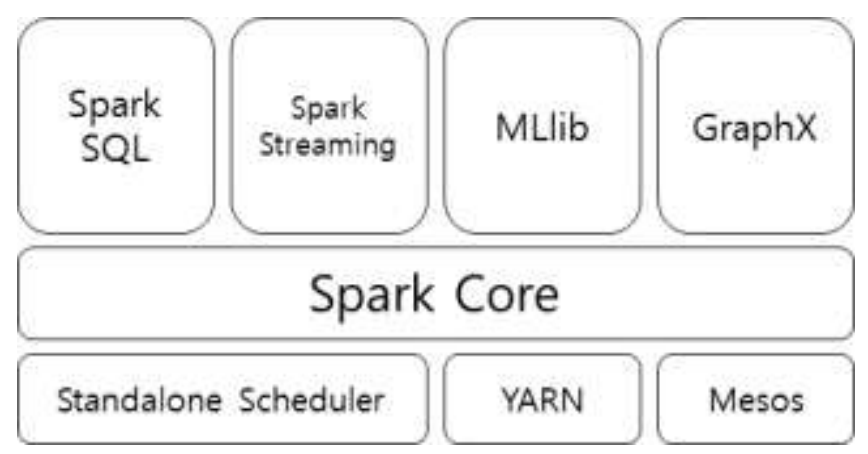

Figure 2. Structure of Apache spark stack

Figure 2 shows the structure of the Apache spark stack. At the infrastructure level, there is a standalone Scheduler, YARN, and Mesos for spark activation. Apache Spark is a memory-based distributed cluster environment, so Spark Core goes up on above the scheduler. The Spark Library layer, then uses the Spark Core to run each library whose purpose is specific to the function. The Library layer provides four submodules (SQL, MLlib, GraphX, streaming). SQL allows you to handle big data, Spark Streaming plays real-time streaming, MLib takes care of machine running, and GraphX is capable of graph data processing. Each submodule is interoperable and can exchange data with each other.

\section{Big Data}

Big Data is a large-scale repository containing more than a tens of terabytes Orthopedic and Atypical data beyond the capabilities of existing database management tools[5]. Big Data focuses on how it does business, and extracts high value from a wide range of large data at low cost. The three major elements of Big Data are Volume, Velocity, and Variety. Volume corresponds to a large data range of tens of terabytes or more. Because data over tens of terabytes is difficult to store in existing file systems, distributed computing techniques must be approached. Velocity should be able to analyze the data quickly and efficiently in real time. To do this, data mining techniques, machine learning, natural language processing, and pattern recognition techniques should be used. Finally, Variety should be able to divide and process the Orthopedic or Atypical data stored in big data into the Orthopedic, semi-structured, and Atypical.

\section{System Design}

The necessary elements for the proposed system can be divided into three. It is necessary to have flume which can batch process gestures data of user, HDFS which holds gestures data, and spark streaming which can process in real time. The Spark Engine plays a role of learning gestures using data received by Spark Streaming and data stored in HDFS.

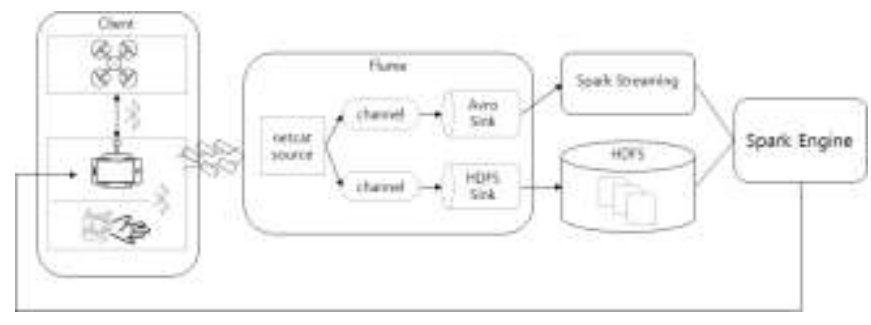

Figure 3. The proposed system design for drone gestures control system using machine learning

Figure 3 is a structure of a machine learning system for learning gestures motions and adding new gestures motions. Generates gestures data using the MYO device, and transfers the data to the controller. The controller sends to the gestures data to the flume at the same time as instructing the operation command with the drone. At this time, the communication between the controller and the flume communicates by the TCP method. For TCP communication, set the source to netcat in the flume agent. The channel consists of two channels. When a channel is configured as one, it is transmitted to either the Avro Sink or the HDFS Sink. But, the proposed system uses two channels to create a new learning model using real-time learning model and stored data. One channel stores the gestures operation in HDFS through the HDFS Sink, and the other channel transmits to Spark Streaming using the Avro Sink. In Spark Engine, gestures data stored in HDFS can be used to increase recognition rate by learning gestures motion in existing model. Also, by using Spark Streaming, gestures data can be learned in model by receiving gestures data transmitted in real time. If the user adds a new gestures, it will behave in the same way as above, and the Spark Engine will perform a new motion-addition algorithm to reflect the new gestures motion in the model. The Spark Engine sends the newly learned model back to the controller, and the controller erases the existing model and reflects the new model.

The advantage of this proposed system structure is that the drone does not need to update the embedded system. The controller changes the learning model in a simple way, recognizes the gestures motion by the changed model, and commands the drone. You can also add custom gestures without extra cost by creating additional UI for the new actions in the controller. This ensures scalability for a variety of gestures.

\section{Iv. Future Work}

In this paper, we propose a learning gestures operation method by building a machine learning system to solve the problem of gestures motion recognition rate of drone. By mechanically learning the gestures recognition rate of the drone, the gestures recognition rate can be increased, and a 
new gestures operation can be added to add a gestures that was not existing.

In future work, we will build a proposed system to confirm that the recognition rate of the gestures motion is increased, and how the gestures recognition rate increases to some extent. A new gestures motion learning model will predicted reduce the recognition rate of existing gestures motion. We also study how new gestures affect existing gestures and develop algorithms to solve them.

\section{Acknowledgment}

This research was supported by Basic Science Research Program through the National Research Foundation of Korea(NRF) funded by the Ministry of Education(NRF2016R1A6A3A11932892)

\section{References}

[1] Campbell, Lee W. et al. "Invariant features for 3-D gesture recognition." Automatic Face and Gesture Recognition, 1996., Proceedings of the Second International Conference on. IEEE, 1996.

[2] Utsumi, Akira, Nobuji Tetsutani, and Seiji Igi. "Hand detection and tracking using pixel value distribution model for multiple-camerabased gesture interactions." Knowledge Media Networking, 2002 Proceedings. IEEE Workshop on. IEEE, 2002.

[3] Liu, Xiufeng, Nadeem Iftikhar, and Xike Xie. "Survey of real-time processing systems for big data." Proceedings of the 18th International Database Engineering \& Applications Symposium. ACM, 2014.

[4] Fu, Jian, Junwei Sun, and Kaiyuan Wang. "SPARK - A Big Data Processing Platform for Machine Learning." Industrial InformaticsComputing Technology, Intelligent Technology, Industrial Information Integration (ICIICII), 2016 International Conference on. IEEE, 2016.

[5] Wu, Xindong, et al. "Data mining with big data." ieee transactions on knowledge and data engineering 26.1 (2014): 97-107.

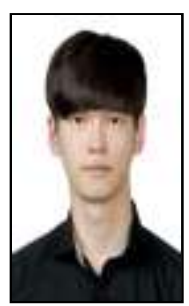

Jang Yeol Lee received the B.S degree in the Information Communication from Korea Nazarene University, Korea, in

2017. Currently, he is working for M.S. in Department of Computer Science and

Engineering at Sungkyunkwan University, Korea. His research interests include Machine learning and Big data.

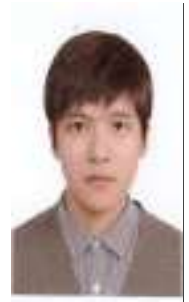

Earl Kim received the B.S degree in the Electronic Engineering from Kyung-Hee University, Korea, in 2015. Currently, he is working for M.S. in Department of Computer Science and Engineering at Sungkyunkwan University, Korea. His research interests include Machine learning and Big data.

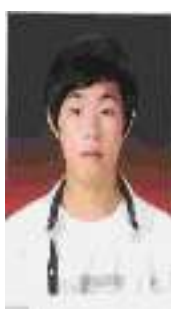

Jun Heon Kim received the B.S degree in the Information \& Communication

Engineering from Sung-Kyul University, Korea, in 2016

Currently, he is working for M.S. in

Department of Computer Science and

Engineering at Sungkyunkwan University, Korea.

His research interests include Machine learning and Big data.

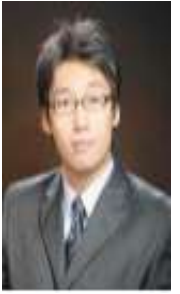

ChoonSung Nam is currently a researcher in Sungkyunkwan University College of Information \& Communication Engineering. He got his Ph.D degree from Sungkyukwan University in August, 2011. his Master's degree from SoongSil

University, and his Bachelor's degree from SangMyung Univeristy, Korea. Dr. Nam is the authoer co-authoer of over 20 papers in sci(e) journals, domestic journals and confereneces. His interested area is UAVs, Machine-learning and IoT.

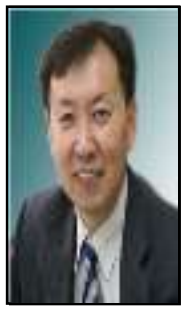

Dong Ryeol Shin received the B.S., M.S., and Ph.D. degrees in Electrical

Engineering from the Sungkyunkwan

University in 1980, the Korea Advanced Institute of Science and Technology (KAIST) in 1982, and the Georgia Institute of Technology in 1992,

respectively. During 1992-1994, he had worked for Samsung Data Systems,

Korea, where he was involved in the research of Intelligent Transportation

Systems. Since 1994, he has been with the Department of Computer Science and

Engineering at Sungkyunkwan University where he is currently a Professor in

Network Research Group. His current research interests lie in the areas of mobile network, ubiquitous computing, cloud computing, and bioinformatics. And he is actively involved in the security of vehicular area networks, and the implementation and analysis of big data platform, applicable to 3D image processing of robotic arms. 